\title{
OXOCARBONOS, PSEUDO-OXOCARBONOS E ESQUARAÍNAS
}

Vanessa E. de Oliveira, Renata Dinize Luiz Fernando C. de Oliveira*

Departamento de Química, Universidade Federal de Juiz de Fora, Campus Martelos, 36036-900 Juiz de Fora - MG, Brasil

Recebido em 19/11/08; aceito em 27/3/09; publicado na web em 26/8/09

OXOCARBONS, PSEUDO-OXOCARBONS AND SQUARAINES. Oxocarbons ions are cyclic compounds presenting unusual electronic and vibrational properties. These molecules anions possess a high symmetry and degree of electronic delocalization, characteristics that have been discussed in several structural and spectroscopic investigations. Compounds in which one or more of the carbonyl oxygen atoms are replaced by other atoms or groups are called pseudo-oxocarbons. Compounds formed by substitution of the carbonyl groups by nitrogen groups former a new class named squaraines. Specificity the dicyanomethylene groups are interesting because of the possibility of further extension of the electronic delocalization and a new coordination site. These molecules also present interesting coordination properties which make these systems potentially useful in crystal engineering research.

Keywords: oxocarbons; pseudo-oxocarbons; squaraines.

\section{INTRODUÇÃO}

Oxocarbonos são compostos orgânicos formados essencialmente por carbono e oxigênio, apresentam-se como diácidos em sua forma neutra e seus ânions possuem fórmula geral $\mathrm{C}_{n} \mathrm{O}_{n}{ }^{2-}$ onde n varia de 3 a 6. Os ânions croconato $(n=5)$ e rodizonato $(n=6)$ foram sintetizados no século XIX, ${ }^{1}$ o esquarato $(n=4)$ foi sintetizado por Cohen e colaboradores em 1959 e o oxocarbono deltato $(n=3)$ por Eggerding e West em 1976 (Figura 1). ${ }^{2,3}$<smiles>O=C1C(=O)C1=O</smiles>

(a)<smiles>O=c1c(=O)c2c(=O)c(=O)c12</smiles>

(b)<smiles>O=C1C(=O)C(=O)C(=O)C1=O</smiles>

(d)

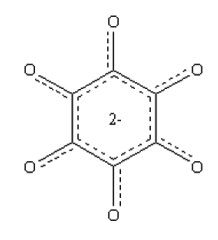

(e)
Figura 1. Representação dos diânions (a) deltato, (b) esquarato, (c) croconato $e(d)$ rodizonato

Os oxocarbonos apresentam características particulares muito interessantes, tais como, estruturas cíclicas planares, elevada simetria molecular, sistemas $\pi$-deslocalizados e espectros eletrônicos singulares por apresentarem o efeito Jahn-Teller. ${ }^{4}$ Uma evidência experimental acerca da estrutura de ressonância destes compostos foi obtida por Hirata e colaboradores através da espectroscopia de absorção no infravermelho. ${ }^{5}$ Nestes estudos foi verificada a ausência da banda referente ao $v(\mathrm{CO})$ que ocorre na região $\sim 1850-1710 \mathrm{~cm}^{-1}$. Em estudos posteriores constatou-se que a variação do tamanho do anel promove uma interessante modificação na deslocalização eletrônica do respectivo oxocarbono: quanto maior o anel menor a deslocalização eletrônica. ${ }^{5}$ Deste ponto de vista , o íon deltato é o único oxocarbono considerado realmente aromático apresentando uma maior estabilidade, devida à maior distribuição de carga, quando comparado aos demais oxocarbonos, e esta estabilidade diminui com o aumento do tamanho do anel. Os demais oxocarbonos são considerados, formalmente, não-aromáticos, porém, apresentando a elevada conjugação eletrônica característica.

*e-mail: luiz.oliveira@ufjf.edu.br
Estas espécies apresentam elevado valor de constante de dissociação, evidenciando a elevada estabilidade conseguida através da ressonância de seus respectivos ânions. Por exemplo, os valores de $\mathrm{pK}_{1}$ e pK 2 para o íon croconato são 0,8 e 2,2, respectivamente, para o íon esquarato são 0,5 e 3,5 e para o rodizonato são 4,3 e 4,7, respectivamente. ${ }^{6}$

A elevada simetria molecular é verificada através de seus espectros eletrônicos, onde o perfil espectroscópico evidencia a presença do efeito Jahn-Teller característico destas espécies (Figura 2). O dupleto observado ocorre devido à dupla degenerescência do primeiro estado eletrônico excitado presente nestas moléculas e afeta seus respectivos espectros vibracionais. ${ }^{4,7}$ Medidas Raman realizadas fora da condição de ressonância apresentam bandas atribuídas a modos não totalmente simétricos com intensidade equivalente ou até mesmo superior aos dos modos totalmente simétricos. ${ }^{8}$ Salienta-se o modo de estiramento da carbonila, modo totalmente simétrico que no espectro Raman aparece como uma banda relativamente pouca intensa quando comparada com outras bandas referentes a modos não totalmente simétricos, tais como o estiramento CC do anel e a deformação do anel. ${ }^{9}$

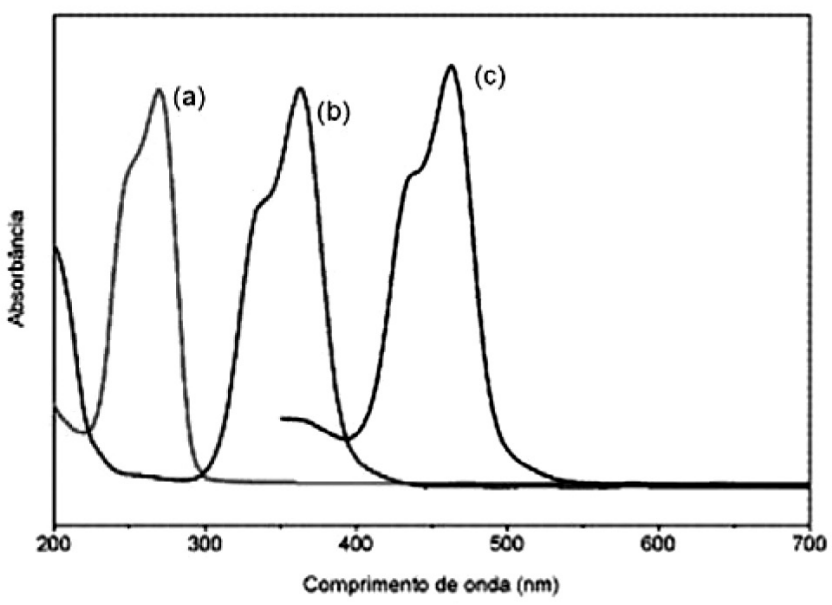

Figura 2. Espectros eletrônicos dos oxocarbonos (a) esquarato, (b) croconato $e$ (c) rodizonato

Os espectros eletrônicos dos sais de potássio para o esquarato, croconato e rodizonato em solução aquosa mostram bandas com altos 
valores de absortividade molar, da ordem de $10^{4} \mathrm{~L} \mathrm{~mol}^{-1} \mathrm{~cm}^{-1}$, além do fato de que estes espectros apresentam um perfil semelhante, com bandas desdobradas. Observa-se que os máximos de absorção são sempre acompanhados de um ombro na região de maior energia com absortividade molar também elevada. Estes íons apresentam fortes absorções nas regiões do ultravioleta e visível, com exceção do derivado deltato que absorve em regiões abaixo de $200 \mathrm{~nm}^{1}$. Para o sal de rodizonato o máximo de absorção ocorre em aproximadamente 500 $\mathrm{nm}$, o croconato absorve em aproximadamente $350 \mathrm{~nm}$ e o esquarato em $250 \mathrm{~nm}$. Observa-se que o deslocamento para regiões de menor energia está diretamente relacionado, dentre outros, com o tamanho do anel oxocarbônico. Este comportamento eletrônico singular explica uma de suas aplicações mais importantes: a utilização destes compostos como sondas do ponto de vista espectroscópico bem como na química de coordenação.

Os espectros de ressonância magnética nuclear de ${ }^{13} \mathrm{C}$ para os diânions como sais de sódio também demonstram esta simplicidade, uma vez que apresentam como único sinal um simpleto em região de campo fraco referente à equivalência de deslocamento químico para os "n" carbonos das carbonilas (entre 204 e 130 ppm). Observase que este deslocamento para o rodizonato está em campo mais fraco (204,2 ppm) que o croconato (189,3 ppm) e estes em campo mais fraco quando comparados ao esquarato $(174,2 \mathrm{ppm}) .^{10}$ Assim, conclui-se que há uma maior deslocalização de carga sobre o anel do esquarato em relação ao croconato e deste em relação ao rodizonato. Características singulares como estas fazem com que estes compostos se tornem de grande interesse quando utilizados como moléculas marcadoras espectroscopicamente.

A simplicidade estrutural demonstrada por estas espécies é evidenciada nos estudos de seus aspectos moleculares, uma vez que sua elevada simetria molecular pode ser verificada através de estudos vibracionais utilizando as técnicas Raman e de absorção no infravermelho, além de cálculos de coordenadas normais e confirmada através de dados obtidos por difração de raios X. ${ }^{11-13} \mathrm{Com}$ base nestes estudos conclui-se que o íon esquarato possui simetria molecular $\mathrm{D}_{4 \mathrm{~h}}$, o croconato $\mathrm{D}_{5 \mathrm{~h}}$ e o rodizonato $\mathrm{D}_{6 \mathrm{~h}}$. Em relação ao deltato também há estudos em relação às suas propriedades espectroscópicas, tanto na forma iônica quanto na forma ácida, onde se concluiu que o deltato possui simetria $\mathrm{D}_{3 \mathrm{~h}}{ }^{14,15}$ Para determinação do grupo pontual do íon esquarato foi feito um estudo analisando o número total de bandas vibracionais teóricas obtidas nas espectroscopias no infravermelho e Raman para cinco diferentes grupos pontuais: $\mathrm{C}_{2 \mathrm{v}}, \mathrm{C}_{2 \mathrm{~h}}, \mathrm{C}_{4 \mathrm{v}}, \mathrm{D}_{2 \mathrm{~d}}$ e $\mathrm{D}_{4 \mathrm{~h}}$ com o número total de bandas obtidas experimentalmente. ${ }^{11}$ Nas Tabelas 1 e 2 estão representadas para os íons esquarato e croconato, respectivamente, a soma total de bandas obtidas através de cálculos feitos a partir de determinado modelo de simetria e os valores encontrados experimentalmente através das diferentes técnicas espectroscópicas indicadas. Com base nestes dados fez-se a correlação entre os dados mais coincidentes e atribuíram-se as simetrias já citadas.

Tabela 1. Número de bandas previstas teoricamente e observadas para o íon esquarato nas técnicas espectroscópicas indicadas

\begin{tabular}{lcccccc}
\hline $\begin{array}{l}\text { Técnica } \\
\text { espectroscópica }\end{array}$ & \multicolumn{5}{c}{ Íon esquarato (teórico) } & $\begin{array}{r}\text { Ín esquarato } \\
\text { (experimental) }\end{array}$ \\
\hline & $\mathrm{C}_{2 \mathrm{v}}$ & $\mathrm{C}_{2 \mathrm{~h}}$ & $\mathrm{C}_{4 \mathrm{v}}$ & $\mathrm{D}_{2 \mathrm{~d}}$ & $\mathrm{D}_{4 \mathrm{~h}}$ & \\
$\begin{array}{l}\text { Raman } \\
\text { polarizado }\end{array}$ & 7 & 5 & 3 & 4 & 2 & 2 \\
Raman total & 18 & 9 & 13 & 13 & 7 & 7 \\
IV & 15 & 9 & 7 & 7 & 4 & 4 \\
\hline
\end{tabular}

Tabela 2. Bandas previstas teoricamente e observadas para o íon croconato nas técnicas espectroscópicas indicadas

\begin{tabular}{lcc}
\hline $\begin{array}{l}\text { Técnica } \\
\text { espectroscópica }\end{array}$ & $\begin{array}{c}\text { Íon croconato } \\
\text { (teórico) }\end{array}$ & $\begin{array}{c}\text { Íon croconato } \\
\text { (experimental) }\end{array}$ \\
\hline Raman polarizado & $\mathrm{D}_{5 \mathrm{~h}}$ & 2 \\
Raman total & 2 & 5 \\
IV & 7 & 3 ou 4 \\
\hline
\end{tabular}

Em 1964 foi determinada a estrutura cristalina para o esquarato de potássio mono-hidratado, confirmando a simetria $\mathrm{D}_{4 \mathrm{~h}} \cdot{ }^{12} \mathrm{~A}$ confirmação da simetria $\mathrm{D}_{5 \mathrm{~h}}$ para o croconato foi feita através da determinação da estrutura cristalina do croconato de potássio, em $1963 .{ }^{13}$

Do ponto de vista eletroquímico os íons oxocarbonos foram estudados por Patton e West; neste trabalho os autores realizaram a síntese eletroquímica dos radicais-ânions para os oxocarbonos esquarato, croconato e rodizonato, em diclorometano. ${ }^{16} \mathrm{~A}$ caracterização foi feita utilizando a técnica de ressonância paramagnética de elétron (EPR). Carré, Fabre e colaboradores obtiveram os espectros eletrônicos para estes radicais-diânions sendo que neste estudo os mesmos foram gerados eletroquimicamente em dimetilformamida. ${ }^{17,18}$ Nestes trabalhos os autores estudaram a oxidação eletroquímica dos ácidos oxocarbônicos utilizando eletrodos de platina em solução de ácido perclórico e propuseram que o mecanismo para o processo oxidativo ocorre em duas etapas de transferência de carga dos compostos na superfície do eletrodo, seguido de dessorção do produto de oxidação que sofre, em seguida, uma reação de hidratação no eletrodo. ${ }^{19}$

Os íons oxocarbonos são muito empregados como fotorreceptores, compostos orgânicos semicondutores e em materiais com propriedades óticas não-lineares. ${ }^{20}$ Sais de rodizonato são amplamente empregados como reagente analítico, por exemplo, na identificação de traços de chumbo em perícia criminal, em análises de rádio em águas doces, além de identificar ferro (II) e bário. ${ }^{21} \mathrm{O}$ rodizonato também atrai bastante interesse devido às suas propriedades luminescentes. ${ }^{21,22}$ $\mathrm{O}$ ácido esquárico é muito utilizado como matéria-prima de produtos utilizados em processos bioquímicos e fotocondutivos. ${ }^{22} \mathrm{~A}$ base conjugada do ácido esquárico tem uma aplicação muito interessante como mimetizador eletrostático de grupos carregados negativamente muito comuns em sistemas biológicos. ${ }^{23}$

O interesse pela família dos oxocarbonos não está somente relacionado à sua considerável simetria molecular e características espectroscópicas, mas também em sua importância na química de coordenação através da complexação com variados metais de transição em uma área promissora conhecida como "Química Supramolecular". Mais recentemente, o interesse está concentrado na exploração principalmente dos diânions croconato e esquarato como ligantes na construção de versáteis arquiteturas de coordenação polimérica, uma vez que estes possuem vários sítios disponíveis para a coordenação com o metal. O diânion esquarato tem sido muito empregado como ligante polifuncional podendo estender a cadeia de várias formas, seja através de ligação de hidrogênio ou através de interações $\pi-\pi$, além de também ser utilizado como ligante em ponte com vários modos de ligação (pontes $\mu_{1}$ a $\mu_{6}$ ) construindo redes inéditas e cada vez mais extensas. ${ }^{24,25}$

As características de ligação do croconato com metais de transição também têm recebido muita atenção e os resultados revelam que este oxocarbono pode se coordenar ao íon metálico de várias maneiras. ${ }^{26}$ $\mathrm{O}$ diânion croconato também funciona como um excelente ligante em ponte na construção de polímeros de coordenação com interessante relação metal-ligante e propriedades magnéticas, devido, principalmente, aos seus versáteis modos de coordenação. ${ }^{27}$ É esta preocupação com a química e com as interações existentes além da molécula que caracteriza 
a química supramolecular e lhe dá a notada importância que vem tendo este novo ramo da química. Sendo os oxocarbonos moléculas quimicamente tão especiais, estes íons estão sendo largamente utilizados para a investigação destas interações e para um melhor conhecimento acerca das propriedades destes íons e respectivos metais, além da predição de novas e interessantes particularidades do sistema como um todo. São estas propriedades que irão determinar a possibilidade de uso de determinado composto no desenvolvimento de um material em especial.

\section{OXOCARBONOS E QUÍMICA DE COORDENAÇÃO}

Nos últimos anos houve um aumento no número de estudos envolvendo estes compostos orgânicos, com interesse em investigar estas interações metal-ligante. ${ }^{21,28} \mathrm{O}$ grupo de pesquisa em espectroscopia molecular do qual este trabalho faz parte tem grande interesse na investigação desta classe de compostos dianiônicos com indiscutíveis particularidades espectroscópicas. Os primeiros trabalhos desenvolvidos basearam-se na tentativa de complexação destes compostos com metais de transição, procurando formar compostos do tipo sanduíche. ${ }^{3,29} \mathrm{O}$ interesse seria a investigação das diversas geometrias de coordenação obtidas e suas características espectroscópicas, buscando estudar a relação e a interação entre as espécies.

Oxocarbonos são compostos extremamente importantes na química de coordenação, ressaltando as várias possibilidades de coordenação e as diversas geometrias em que estas espécies podem ser encontrar, alterando suas propriedades estruturais. , $30,31^{2}$

Nos últimos anos, observa-se na literatura um grande interesse na investigação das propriedades cristalográficas de estruturas supramoleculares envolvendo íons oxocarbonos, devido a sua grande utilização na engenharia cristalina como blocos construtores, principalmente os oxocarbonos esquarato e croconato. ${ }^{32-36}$ São conhecidos, também, compostos de metais de transição envolvendo estes íons, uma vez que possuem interessantes propriedades magnéticas e óptico-eletrônicas. ${ }^{37}$

O ácido esquárico e suas espécies desprotonadas (íons hidrogenoesquarato e esquarato) são de interesse especial como unidades geradoras de supermoléculas em química supramolecular. ${ }^{20,37}$ É amplamente conhecido como um ligante capaz de propagar uma variedade de interações magnéticas exibindo diferentes sítios para a coordenação (Figura 3), ligando-se na forma de quelato 1,2-bidentado, em ponte através das posições 1,2-bis(monodentado) e 1,3-bis(monodentado) ou, ainda, através dos quatro átomos de oxigênio da sua estrutura. ${ }^{37-43}$ Variando o metal ou a metodologia de síntese podem ser gerados compostos com interessantes propriedades eletrônicas e vibracionais. . $^{12,40,44}$ Este íon pode ser usado na produção de variadas topologias de rede em química supramolecular através de fortes ligações de hidrogênio, ${ }^{45}$ possuindo características interessantes como as das zeólitas, forte luminescência e até magnetismo, no caso de complexos metálicos. ${ }^{46}$

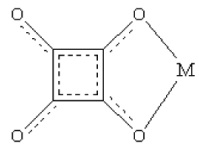

(a)

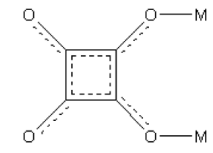

(b)

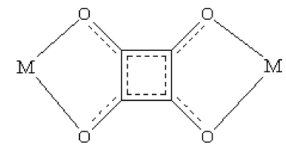

(e)
Figura 3. Modos de coordenação do íon esquarato: (a) bidentado, (b) cis bismonodentado, (c) trans-bismonodentado, (d) tetra-monodentado e (e) bis-bidentado

\section{Pseudo-oxocarbonos}

Nas últimas décadas, as reatividades dos íons oxocarbonos foram investigadas em detalhes e, como consequência, vários derivados foram sintetizados; nestes trabalhos vários estudos foram feitos a respeito da reatividade dos mesmos, além de uma infinidade de possíveis compostos que podem ser usados com o objetivo de promover a substituição dos átomos de oxigênio dos precursores. ${ }^{47,48} \mathrm{~A}$ substituição completa ou parcial dos átomos de oxigênio carbonílicos por diferentes grupos substituintes leva à formação de compostos denominados pseudo-oxocarbonos. ${ }^{40}$ Cada espécie pode ser considerada como originada de um precursor oxocarbônico, na qual um ou mais átomos de oxigênio são trocados por outros átomos ou grupos funcionais. Porém, poucos destes derivados têm sido explorados em maiores detalhes do ponto de vista espectroscópico.

Espécies oxocarbônicas totais ou parcialmente substituídas por átomos de fósforo, selênio ou grupos nitrogenados são exemplos dos chamados pseudo-oxocarbonos. ${ }^{41,42}$ Dentre estes compostos encontram-se também os derivados de enxofre, onde a natureza do grupo substituinte é tal que a deslocalização eletrônica presente é substancialmente estendida, atraindo interesse, principalmente no contexto de materiais óticos com propriedades não-lineares. ${ }^{20} \mathrm{Os}$ pseudo-oxocarbonos mais estudados para os respectivos oxocarbonos estão esquematizados nas Figuras 4 a 7.

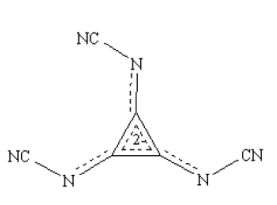

(a)

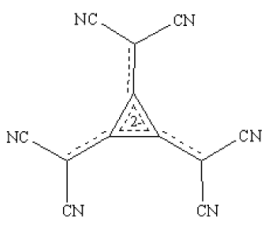

(b)

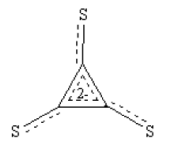

(c)
Figura 4. Pseudo-oxocarbonos mais estudados para o íon deltato: (a) ion tris(cianoimino) deltato, (b) íon tris(dicianometileno) deltato e (c) ion tritiodeltato

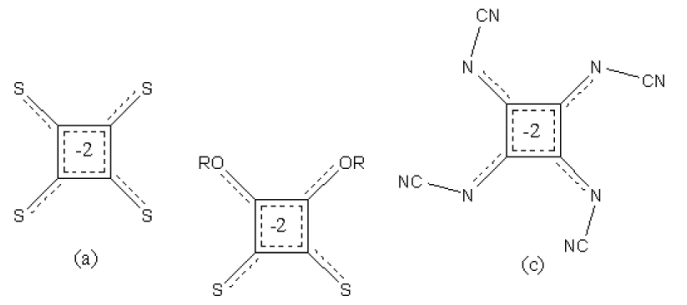

(b)

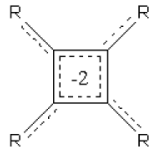

(d)
Figura 5. Pseudo-oxocarbonos mais estudados para o íon esquarato: (a) ion tetratioesquarato, (b) íon 1,2 ditioesquarato, (c) ion tetra(cianoimino) esquarato e (d) íons carboesquaratos

Os pseudo-oxocarbonos derivados do diânion croconato mais estudado são o croconato violeta [3,5-bis(dicianometileno)ciclopentano-1,2,4-trionato] e o croconato azul [2,4,5-tris(dicianometileno) ciclopentano-1,3-dionato]. ${ }^{15-19}$ Estes compostos atraem bastante interesse devido ao seu reversível caráter eletroquímico; ${ }^{20,21}$ fortes absorções na região do visível justificando sua intensa coloração (absortividade molar da ordem de $10^{5} \mathrm{~L} \mathrm{~mol}^{-1} \mathrm{~cm}^{-1}$ ), expressiva extensão do sistema eletrônico $\pi$ e típicas propriedades semicondutoras. ${ }^{22,23}$ Estes compostos apresentam ainda propriedades importantes para o desenvolvimento de materiais com propriedades interessantes em estudos fotofísicos, por exemplo, conversão de energia solar, materiais eletrocrômicos, dentre outros. ${ }^{6,9} \mathrm{O}$ croconato violeta exibe essencial- 
<smiles></smiles>

(a)

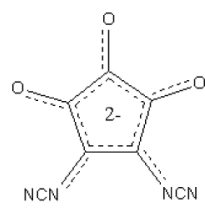

(b)<smiles></smiles>

(d)

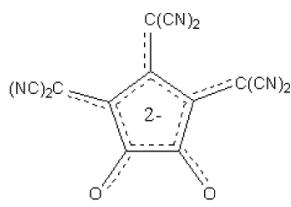

(c)

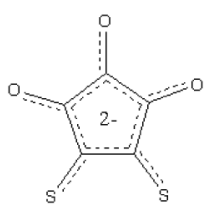

(e)

Figura 6. Pseudo-oxocarbonos mais estudados para o íon croconato: (a) íon 1,3 bis(cianoimino) croconato, (b) íon 1,2 bis(cianoimino) croconato, (c) ion 1,2,3 tris(dicianometileno) croconato, (d) ion 1,3 bis(dicianometileno) croconato e (e) íon 1,2 ditiocroconato<smiles>NC1=C(N)C(=O)C(N)=C(N)C1=O</smiles>

(a)<smiles>S=C1C(=S)C(=S)C(=S)C(=S)C1=S</smiles>

(b)

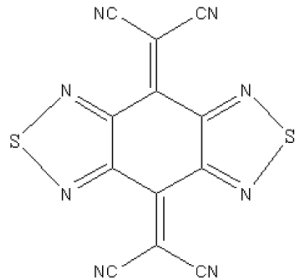

(c)

Figura 7. Pseudo-oxocarbonos mais estudados para o ín rodizonato: (a) tetra (amino)-p-benzoquinona, (b) ion hexatio rodizonato e (c) tetracianoquinodimetano

mente dois diferentes modos de coordenação, através dos átomos de oxigênio vicinal como quelante ou através dos átomos de nitrogênio do grupo dicianometileno no modo em ponte. ${ }^{49,50}$ Alguns complexos com o croconato violeta apresentam interações intramoleculares em que o metal pode transmitir propriedades eletrônicas para aplicações eletrocatalíticas ou eletrocrômicas. ${ }^{51}$ Mais recentemente, foi observado que este ligante versátil pode também atuar como um ligante em ponte conectando três cátion de $\mathrm{Fe}$ (II) através dos átomos de oxigênio vicinais e um átomo de nitrogênio de cada grupo dicianometileno para formar um polímero de coordenação $1 \mathrm{D} .^{52}$

Os oxocarbonos croconato violeta, croconato azul, croconato de lítio e 1,2-bis(dicianometileno)esquarato de potássio são altamente solúveis em água e em muitos solventes não-aquosos, restringindo sua utilização. ${ }^{53}$ Mas este fato pode ser contornado para o croconato violeta, por exemplo, incorporado-o em um filme protonado de poli(4vinilpiridina) que, após tratamento adequado, exibe interessante propriedade eletrocatalítica. ${ }^{54}$

No caso do íon esquarato (ou na forma ácida: 1,2-di-hidroxiciclobuteno-3,4-diona), além dos pseudo-oxocarbonos citados, numerosos derivados têm sido preparados com cadeias carbônicas, nitrogênio, enxofre e selênio como substituintes, com inúmeras aplicações nas mais diferentes áreas. ${ }^{43,55} \mathrm{~A}$ síntese de espécies de coordenação com os pseudo-oxocarbonos tioderivados demonstra o interesse no entendimento das características e comportamento químico para, por exemplo, desenvolver materiais com dimensão reduzida e que demonstrem propriedades metálicas ou semicondutoras. ${ }^{42}$

O ácido esquárico por si só possui uma estrutura muito interessante, portanto, a modificação desta estrutura primitiva pode trazer novas propriedades físico-químicas, bem como a intensificação de

outras características pré-existentes, como o aumento da deslocalização eletrônica.

\section{ESQUARAÍNAS}

Apesar do conceito de esquaraína ser polêmico, vários autores convergem quanto ao fato de serem esquaraínas verdadeiras os produtos que apresentam um ou mais grupos nitrogenados em substituição aos átomos de oxigênio do íon esquarato, esta nova classe de compostos está incluída em uma interessante família de fotocondutores orgânicos (Figura 8).

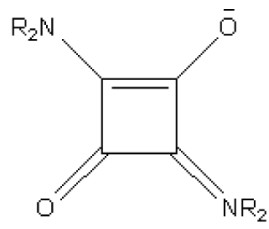

Figura 8. Esquaraínas 1,3 substituídas por grupos nitrogenados

O primeiro produto desta classe de compostos foi preparado em 1965 em uma reação entre o ácido esquárico e pirrois, formando produtos de condensação intensamente coloridos (Figura 9). ${ }^{56} \mathrm{Um}$ estudo realizado por nosso grupo de pesquisa remete-se à síntese de uma interessante esquaraína obtida com o resorcinol (hidroxifenil). Atenta-se ao fato de que este composto não apresenta átomos de nitrogênio no ligante, reafirmando que não existe na literatura um consenso acerca do conceito de esquaraínas. ${ }^{57}$

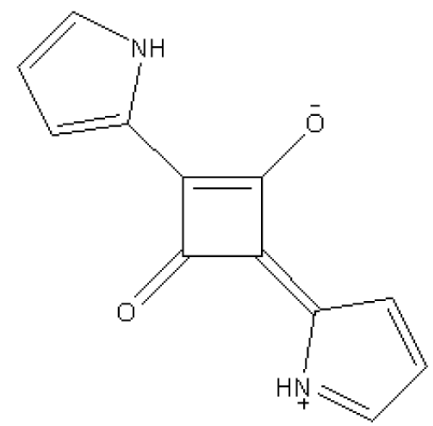

Figura 9. Produto da reação entre pirrol e ácido esquárico

São geralmente preparados pela condensação de compostos aromáticos ou heteroaromáticos ricos em elétrons como N,N-dialquilanilinas, benzotiois, fenois, azulenos e pirrois com ácido esquárico. Assim, um enorme número de sistemas tem sido sintetizado com a variação do sistema doador, havendo várias possibilidades para design de uma infinidade de esquaraínas com características particulares. No estado sólido vários destes compostos exibem uma banda de absorção com significativo coeficiente de absorção, devido à autoagregação que deve ser controlada a fim de melhorar a eficiência do dispositivo de interesse..$^{58}$

Cálculos semiempíricos de orbitais moleculares mostram que ambos os estados fundamental e excitado das esquaraínas são estados com transferência de carga intramolecular do tipo doador-receptordoador (D-A-D). ${ }^{59}$ É uma classe de compostos com estrutura zwiteriônica estabilizada por ressonância. Esquaraínas típicas contêm um anel de quatro membros central deficiente em elétrons e dois grupos elétron-doadores. Como monômeros em solução, estes compostos absorvem fortemente na região do visível em regiões acima de 600 $\mathrm{nm}$ com altos valores de absortividade molar $\left(\varepsilon>10^{5} \mathrm{~L} \mathrm{~mol}^{-1} \mathrm{~cm}^{-1}\right)$ e intensa emissão fluorescente com pequeno deslocamento Stokes, além disso são fotoestáveis. ${ }^{58,59} \mathrm{~A}$ maioria dos estudos está concentrada 
nas esquaraínas do tipo 1,3-substituídas, cercado por dois grupos doadores de elétrons na conformação $D-A-D$ que permite elevado grau de transferência de carga intramolecular. ${ }^{60}$ Geralmente, o átomo de nitrogênio está diretamente ligado ao anel (Figura 10). ${ }^{47,61-63}$ Estas esquaraínas dissubstituídas mostram altos coeficientes de absorção na região do vermelho (maior que $2 \times 10^{5} \mathrm{~L} \mathrm{~mol}^{-1} \mathrm{~cm}^{-1}$ ) e demonstram considerável fotoestabilidade. ${ }^{64}$ Estes compostos apresentam propriedades interessantes, tais como fotocondutividade e elevada absorção no vermelho ou infravermelho próximo. Propriedades estas que possibilitam a sua aplicação em xerografia ou na confecção de lasers de diodo. Estudos preliminares demonstram que esquaraínas não-centrossimétricas apresentam maior probabilidade de desenvolverem propriedades ópticas não-lineares. ${ }^{65}$
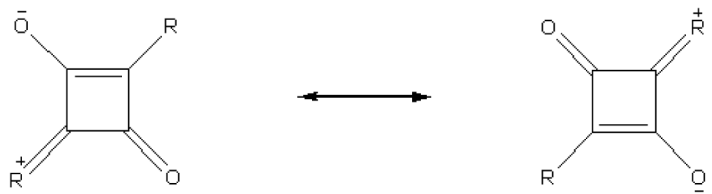

(a)

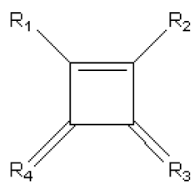

(b)

Figura 10. Representação geral das esquaraínas (a) ressonância de esquaraínas trans substituídas e (b) esquema geral (R: grupos contendo nitrogênio)

Esquaraínas com substituições no anel oxocarbônico que absorvem e emitem no vermelho e infravermelho próximo também têm sido desenvolvidas. ${ }^{66}$ Estes sistemas têm vantagem quando comparados às chamadas Cy5-NHS convencionais (cadeia aberta). Como exemplo, estes compostos podem ser excitados não somente com lasers vermelhos, mas igualmente com um laser azul ou com diodos luminescentes. Devido às suas propriedades espectrais e fotofísicas favoráveis, estes compostos são particularmente úteis para aplicações biomédicas, principalmente devido à fluorescência destes compostos. ${ }^{66}$

Alguns estudos têm sido feitos no sentido de desenvolver poliesquaraínas chamadas esquaraínas de absorção de amplo espectro. Embora as propriedades óticas destes sistemas sejam ideais para uma variedade de materiais óptico-eletrônicos encontra-se uma significativa dificuldade na solubilização e processamento destes compostos. ${ }^{67}$

Algumas esquaraínas estão sendo sintetizadas com interesse no desenvolvimento de quimiossensores para a detecção seletiva de analitos específicos em sistemas que vão desde os químicos aos biológicos. ${ }^{68}$ Como exemplo, tem-se a detecção seletiva de cátions biologicamente importantes, tais como, $\mathrm{Na}^{+}, \mathrm{K}^{+}, \mathrm{Mg}^{2+}$ e $\mathrm{Ca}^{2+}$. É neste sentido que as esquaraínas se tornam importantes devido às suas propriedades ópticas peculiares. Nestes trabalhos têm-se diferentes cromóforos ligados a sistemas do tipo poliéteres pseudocíclicos utilizados para captura de cátions. ${ }^{69}$

Esquaraínas apresentam sabidamente forte absorção e emissão na região do infravermelho próximo. Devido à natureza da transferência de carga intermolecular destas transições, que são altamente sensíveis à mudança de substituintes bem como do solvente,,$^{70}$ estes compostos se tornam ideais para o design de novos fluoroionoforos. Ionóforos covalentemente ligados a redox-ativos (resíduos com habilidade em sofrer processos redox-reversíveis) são muito importantes como sondas de sensibilidade potencial para íons metálicos, bem como para o transporte de íons metálicos através das membranas. ${ }^{71,72}$

$\mathrm{O}$ estudo destas esquaraínas cresceu muito nos últimos anos, principalmente no desenvolvimento de materiais fluorescentes, ou ainda na aplicação em baterias fotoeletroquímicas, em elementos de conversão fotoelétrica, ${ }^{73}$ substrato para células fotovoltaicas, fotorreceptores e fotocondutores. ${ }^{74}$ Estas aplicações em potencial fazem com que haja um crescente interesse por este tipo de composto, tanto no desenvolvimento das sínteses quanto no estudo de suas propriedades físico-químicas. Dependendo do tipo de substituinte podem-se obter compostos com comportamento bastante diferenciado, fato este que implica em grande variedade de compostos disponíveis na literatura. ${ }^{55,59,61-65,74} \mathrm{Em}$ nosso grupo de pesquisa algumas esquaraínas estão sendo estudadas, ${ }^{20,73}$ tendo como grupos substituintes a anilina e alguns compostos alifáticos (Figura 11).

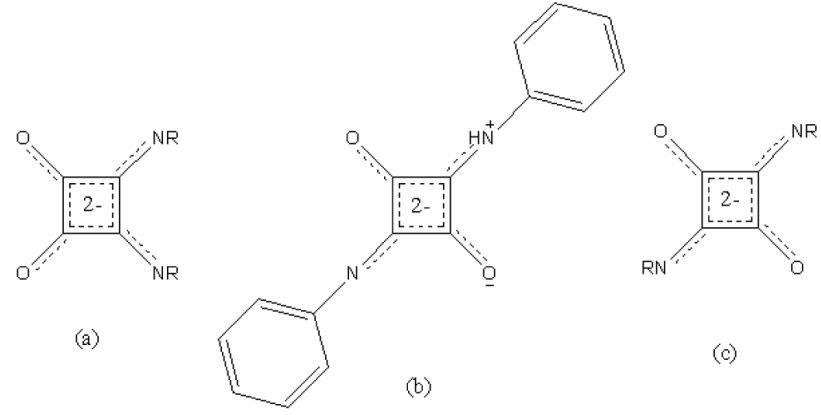

Figura 11. Esquaraínas 1,2 e 1,3 substituídas por grupos nitrogenados estudadas por nosso grupo

Uma gama de compostos que tem sido muito utilizada principalmente na técnica de espalhamento Raman Ressonante aplicado à superfície (SERRS) são as esquaraínas anilino modificadas. ${ }^{75} \mathrm{~A}$ estrutura geral (Figura 12) mostra o sistema quadrupolar $D-A-D$ que é caracterizado pela ciclobutenodiona $\left(\mathrm{C}_{4} \mathrm{O}_{2}\right)$ em ponte como um sistema deficiente de elétron. A estrutura total pode ser representada em um grande número de formas e a carga é distribuída por toda a molécula de forma homogênea. As estruturas desta classe de materiais têm sido objeto de vários estudos teóricos, principalmente para examinar as incomuns propriedades ópticas não-lineares. ${ }^{76}$

D-A-D-A-D

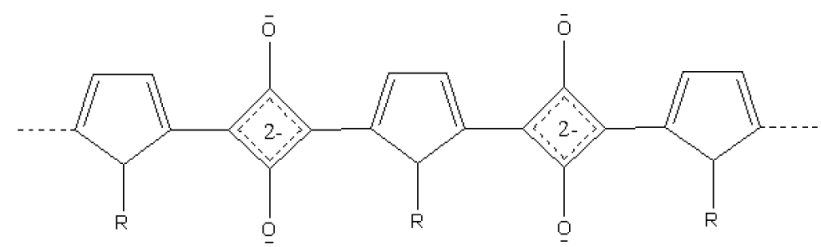

Figura 12. Estrutura geral de um sistema quadrupolar doador-receptordoador caracterizado pela ciclobutenodiona $\left(\mathrm{C}_{4} \mathrm{O}_{2}\right)$ em ponte como um sistema deficiente de elétron

Em particular, a síntese de compostos aniônicos derivados do ácido esquárico que apresentam uma ou mais substituições por grupos com elétrons $\pi$-deslocalizados, como os grupos dicianometilenos, é de bastante interesse. A introdução de grupos com elétrons p aumenta a extensão da forte deslocalização eletrônica pré-existente em um sistema do tipo $D-A-D$ de elétrons, gerando compostos intensamente coloridos, com fluorescência característica. ${ }^{1}$ 
Encontra-se na literatura um grande número de estudos referentes à síntese de compostos substituídos por estes grupos (dicianometileno). ${ }^{1,77-79} \mathrm{~A}$ partir da substituição de um ou mais átomos de oxigênio por outros grupos, como exemplo, a malononitrila (Figura 13), pode-se obter esquaraínas bastante interessantes (Figura 14). A malononitrila é um composto bastante reativo, sistematicamente empregado em reações de substituição em sistemas oxocarbonos, especialmente nos íons croconato e no esquarato ${ }^{80}$

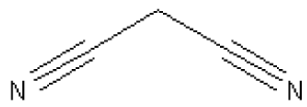

Figura 13. Estrutura molecular da malononitrila

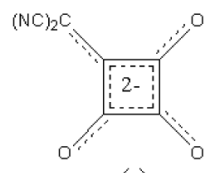

(a)

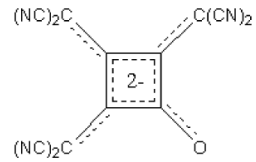

(d)

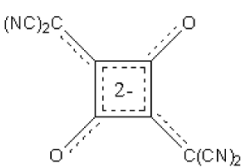

(b)

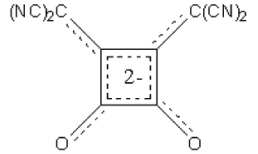

(c)

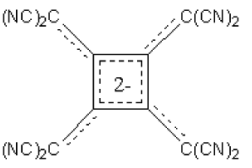

(e)

Figura 14. Estruturas moleculares de esquaraínas substituídas: (a) íon mono (dicianometileno) esquarato; (b) íon trans bis(dicianometileno) esquarato; (c) ion cis bis(dicianometileno) esquarato; (d) ion tris (dicianometileno) esquarato e (e) íon tetra (dicianometileno) esquarato

Em um artigo de revisão, Gerechet e colaboradores fazem uma síntese de todas as rotas utilizadas para a obtenção destes compostos descritas na literatura.$^{45}$ Neste trabalho, além do estudo da reatividade frente às várias possibilidades, os autores também reportam estudos espectroscópicos em técnicas como absorção no infravermelho e ressonância magnética nuclear de ${ }^{13} \mathrm{C}$. Porém, existem pouquíssimos estudos em que as várias espectroscopias são discutidas em conjunto, excetuando recentes trabalhos realizados por nosso grupo de pesquisa. ${ }^{81}$

Lunelli e colaboradores reportam a estrutura cristalina do 1,3 bis(dicianometileno) esquarato de sódio e cálculos computacionais das frequências vibracionais fundamentais para o respectivo diânion..$^{77}$ Neste trabalho, Lunelli reporta a estrutura cristalina do 1,3 bis(dicianometileno) esquarato de sódio tetra-hidratado, cuja geometria do ânion livre tem simetria $\mathrm{D}_{2 \mathrm{~h}}$ com estrutura essencialmente planar (desvios de aproximadamente $0,05 \AA$ ). As moléculas de água são fundamentais para a arquitetura do cristal, uma vez que estão coordenadas aos cátions sódio, além do fato de proporcionarem estabilidade ao cristal com fortes ligações de hidrogênio. De posse das distâncias das ligações chega-se à conclusão de que a estrutura representada na Figura 15 representa a menor perturbação nas ligações evidenciadas pelas formas canônicas com carga negativa localizada nos átomos mais eletronegativos da molécula.

A estrutura cristalina da esquaraína 1,2 substituída por grupos dicianometileno de sódio com meia molécula de dioxano e quatro moléculas de água também é reportada na literatura. ${ }^{78} \mathrm{O}$ anel encontra-se planar com pequenos desvios, as distâncias de ligações dos átomos de carbono pertencentes ao anel podem ser consideradas iguais com um valor de $1,422 \AA$, o que indica um significante caráter residual de dupla ligação. Medidas de ESR (Electron Spin Resonance

or Electron Paramagnetic Resonance) confirmam que a estrutura do 1,2-bis(dicianometileno) esquarato possui uma extensão do caráter de elétrons $\mathrm{p} .{ }^{78} \mathrm{~A}$ molécula de dioxano está localizada sobre um centro de inversão, tem uma conformação na forma de cadeira e é um componente essencial para o crescimento do cristal. As várias camadas são estendidas por fortes ligações de hidrogênio e duas unidades do diânion estão relacionadas entre si por um centro de inversão.

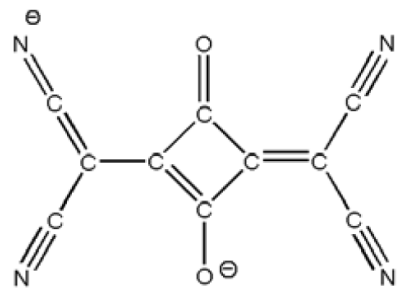

Figura 15. Forma canônica que indica a concentração de carga negativa sobre os elementos mais eletronegativos do íon 1,3-bis(dicianometileno) esquarato

Compostos com metais de transição são bastante raros na literatura, como exemplo, para a esquaraína cis substituída apresenta a estrutura cristalina de um complexo com $\mathrm{Cu}$ (I) ${ }^{79}$ Galibert e colaboradores sintetizaram um complexo de $\mathrm{Cu}$ (II) com a esquaraína 1,3. ${ }^{65}$ $\mathrm{O}$ anel do pseudo-oxocarbono é planar. Entretanto, os desvios dos grupos ciano do melhor plano formado pelo anel são significativos e contribuem para uma perda de planaridade no complexo e, esta é acompanhada por uma perda de deslocalização pi no anel.

A estrutura cristalina para o diânion do composto 1,3 bis(dicianometileno) esquarato com duas moléculas de fenilfosfina e duas moléculas de água foi caracterizada por difração de raios X. ${ }^{47}$ Este composto cristaliza-se num sistema triclínico e grupo espacial P1̄.

Com o diânion como ligante, mais dois complexos foram obtidos e caracterizados por difração de raios X, espectroscopia UV-vis e, ainda, por medidas eletroquímicas. ${ }^{62}$ Complexo com $\mathrm{Cu}$ (II) com o diânion mais quatro moléculas de água coordenadas e mais duas de hidratação forma uma cadeia polimérica com os átomos de cobre ligados a dois grupos nitrilas do ligante.

O complexo de cobre (I) com o ligante 1,3-substituído cristalizase no grupo espacial monoclínico $\mathrm{P} 22_{1} / \mathrm{n} .{ }^{47}$ Nesta estrutura quatro moléculas de acetonitrila encontram-se coordenadas e duas moléculas não coordenadas formam uma cadeia polimérica com os átomos de cobre ligados pelos quatro grupos nitrila do ligante. Em solução de acetonitrila, os complexos de cobre se dissociam. Além disso, o $\mathrm{Cu}$ (II) é reduzido a $\mathrm{Cu}$ (I) pelo diânion. A eletroquímica no estado sólido (polímero que reveste o eletrodo) mostra a transição redox dos diferentes compostos.

O único metal de transição utilizado na literatura para complexação com estes diânions 1,2 e 1,3, cis e trans respectivamente, é o átomo de cobre. Em um trabalho recente desenvolvido pelo nosso grupo de pesquisa, mais duas estruturas cristalinas para complexos inéditos com metais de transição foram sintetizadas e caracterizadas por diferentes técnicas espectroscópicas e por difração de raios X de monocristal. ${ }^{81}$ Na Figura 16 estão representadas as estruturas cristalinas obtidas para os compostos de coordenação da esquaraína trans bis(dicianometileno) esquarato para os metais $\mathrm{Zn}$ (II) e Co (II), respectivamente. $\mathrm{O}$ complexo de zinco é isoestrutural do complexo de cobre (II) descrito anteriormente, pertence ao grupo espacial monoclínico $\mathrm{P} 2{ }_{1} / \mathrm{c} .{ }^{82} \mathrm{O}$ complexo com o cobalto pertence ao sistema monoclínico, grupo espacial C2/m; ambos os complexos consistem de cadeias poliméricas com os respectivos metais ligados a dois átomos de nitrogênio de ligantes diferentes. A molécula estende-se em uma estrutura polimérica bidimensional; em uma direção, a cadeia 
se estende através de ligações de hidrogênio e em outra, através da coordenação ligante e metal. Ambos os metais encontram-se coordenados ao átomo de nitrogênio do grupo ciano de forma monodentada e juntamente com moléculas de água apresenta-se em uma geometria octaédrica distorcida. Não se observa a presença de interação pi entre os anéis dos ligantes, uma vez que as distâncias entre os centróides dos respectivos anéis estão acima de 6 Å para ambos os complexos.

(a)

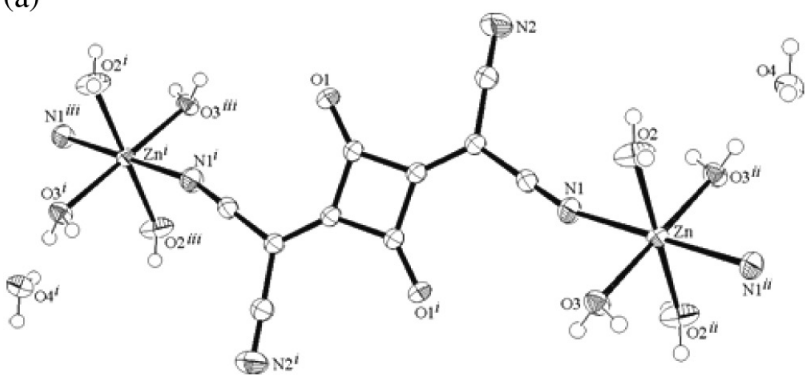

(b)

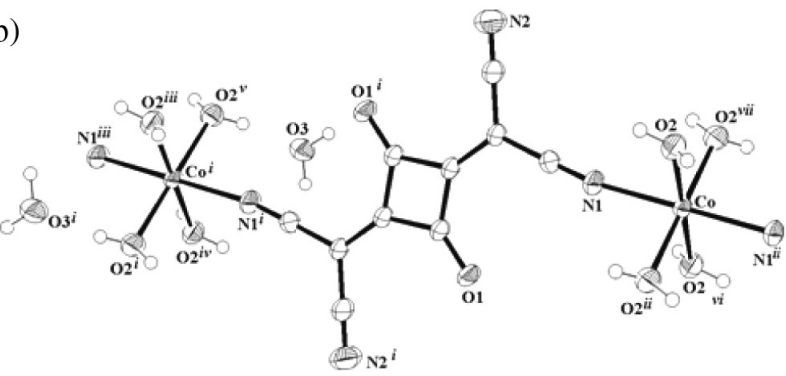

Figura 16. Estruturas cristalinas obtidas para os compostos de coordenação da esquaraína (a) trans bis(dicianometileno) esquarato para os metais zinco (II) $e$ (b) cobalto (II)

Além das duas estruturas cristalinas obtidas neste trabalho, mais seis compostos de coordenação foram sintetizados e caracterizados. ${ }^{67,81}$ Foi feito um estudo espectroscópico comparativo completo envolvendo as esquaraínas 1,3 e 1,2 substituídas por grupos dicianometileno e quatro metais de transição divalentes, dentre eles cobalto, manganês, zinco e níquel. Através dos espectros vibracionais Raman e infravermelho de todos os complexos obtidos com ambos os ligantes, observou-se que estes apresentam o elemento de simetria inversão. A complexação não afeta a simetria molecular (para o caso do diânion trans) e no estado sólido (para o diânion cis).

No espectro eletrônico dos ligantes observa-se que a substituição de átomos de oxigênio por grupos $\left[\mathrm{CC}(\mathrm{CN})_{2}\right]$ resulta em uma substancial deslocalização na energia da transição $\pi \rightarrow \pi^{*}$, devido à introdução de uma nova transição que envolve os grupos substituintes.

A síntese de oxocarbonos substituídos por grupos dicianometileno é interessante uma vez que é possível, dentre outras razões, explorar as modificações espectrais causadas pela modificação da simetria. $\mathrm{O}$ estudo espectroscópico feito em variadas técnicas trouxe informações relevantes do ponto de vista de estabilidade e reatividade. Mas ainda existe uma lacuna no que diz respeito aos mecanismos de síntese, ao estudo espectroscópico e até de estruturas cristalinas destes ligantes com os demais metais de transição. O estudo espectroscópico destas espécies químicas é de grande importância para a compreensão dos aspectos vibracionais e eletrônicos que regem este grupo singular de pseudo-oxocarbonos. Estudar características vibracionais de derivados oxocarbônicos faz-se necessário uma vez que amplia o conhecimento acerca dos próprios precursores.

Em conclusão, as esquaraínas são importantes substâncias orgânicas com propriedades ópticas úteis e de notável interesse para o desenvolvimento de uma variedade de materiais óptico-eletrônicos, sendo consideravelmente susceptíveis a estímulos fotoelétricos. Consequentemente, é razoável concluir que o futuro da química tanto destes compostos quanto a de seus precursores continuará a ser bastante promissor e viável.

\section{AGRADECIMENTOS}

Ao CNPq, CAPES e FAPEMIG pelo apoio financeiro.

\section{REFERÊNCIAS}

1. Seitz, G.; Imming, P.; Chem. Rev. 1992, 92, 1227.

2. Cohen, S.; Lacher, J. R.; Park, J. D.; J. Am. Chem. Soc. 1959, 81, 3480.

3. Eggerding, D.; West, R.; J. Am. Chem. Soc. 1976, 98, 3641.

4. Junqueira, G. M. A.; Rocha, W. R.; de Almeida, W. B.; dos Santos, H. F.; Phys. Chem. Chem. Phys. 2002, 4, 2517.

5. Campos-Vallette, M.; Figueroa, K. A.; Puebla. C.; Spec. Lett. 1988, 21, 303; Yamada, K.; Mizuno, N.; Hirata, Y.; Bull. Chem. Soc. Jpn. 1958, 31,543 .

6. West, R.; Oxocarbons, Academic: London, 1980.

7. Junqueira, G. M. A.; Rocha, W. R.; de Almeida, W. B.; dos Santos, H. F.; J. Mol. Struc. (Theo.) 2005, 719, 31.

8. Santos, P. S.; Sala, O.; Noda, L. K.; Gonçalves, N. S.; Spec. Acta A 2000, $56,1553$.

9. Takahashi, M.; Kaya, K.; Ito, M.; J. Chem. Phys. 1978, 35, 293.

10. Stadeli, W.; Hollenstein, R.; Philipsborn, W.; Helv. Chim. Acta 1977, 60, 948.

11. Ito, M.; West, R.; J. Am. Chem. Soc. 1963, 81, 2580; Santos, P. S.; Amaral, J. H.; de Oliveira, L. F. C.; J. Mol. Struc. 1991, 243, 223.

12. Macintyre, M. M.; Werkema, M. S.; J. Chem. Phys. 1964, 42, 3563.

13. Baezinger, N. C.; Hegenbarth, J. J.; Williams, D. J.; J. Am. Chem. Soc. 1963, 85, 1539.

14. West, R.; Eggerding, D., Perkins, J.; Handy, D.; Tuazon, E. C.; J. Am. Chem. Soc. 1979, 101, 1710.

15. Lautié, A.; Lautié, M.-F.; Novak, A.; Can. J. Chem. 1985, 63, 1394.

16. Patton, E. V.; West, R.; J. Phys. Chem. 1973, 77, 2652.

17. Carré, B.; Paris, J.; Fabre, P. L.; Jourdannaud, S.; Castan, P.; Deguenon, D.; Winner, S.; Bull Soc. Chim. Fr. 1990, 127, 367.

18. Fabre, P. L.; Dumestre, F.; Soula, B.; Galibert, A. M.; Electrochim. Acta 2000, 45, 2697.

19. Sant'Ana, A. C.; Santos, P. S.; Temperini, M. L. A.; J. Electroanal. Chem. 2004, 571, 247.

20. Lopes, J. G. S.; Farani, R. A.; de Oliveira, L. F.; Santos, P. S.; J.Raman Spec. 2004, 35, 131

21. de Oliveira, L. F. C.; Santos, P. S.; J. Mol. Struct. 1992, 269, 85.

22. Brouca-Cabarrecq, C.; Mohanu, C. A.; Millet, P.; Trombe, J. C.; J. Sol. State Chem. 2004, 177, 2575.

23. Onaran, M. B.; Comeau, A. B.; Seto, C. T.; J. Org. Chem. 2005, 70, 10792.

24. Dan, M.; Rao, C. N. R.; Solid State Science 2003, 5, 615; Kurmoo, M.; Kumagai, H.; Chapman, K. W.; Kepert, C. J.; Chem. Commun. 2005, 3012; Chen, Q.; Liu, S.; Zubieta, J., Angew. Chem., Int. Ed. 1990, 29, ???; Khan, M. I.; Chang, Y. D.; Chen, Q.; Salta, J.; Lee, Y. S.; O’Connor, C. J.; Zubieta, J., Inorg. Chem. 1994, 33, 6340; Lee, C. R.; Wang, C. C.; Wang, Y.; Acta. Crystallogr. 1996, B52, 966; Yang, C. H.; Chou, C. M.; Lee, G. H.; Wang, C. C.; Inorg. Chem. Commun. 2003, 6, 135; Wang, C. C.; Yang, C. H.; Tseng, S. M.; Lee, G. H.; Sheu, H. S.; Phyu, K. W.; Inorg. Chim. Acta 2004, 357, 3759; Wang, C. C.; Yang, C. H.; Lee, G. H.; Tsai, H. L.; Eur. J. Inorg. Chem. 2005, 1334; Wang, C. C.; Yang, C. H.; Lee, G. H.; Eur. J. Inorg. Chem. 2006, 820; Hall, L. A.; Williams, D. J.; Menzer, S.; White, A. J. P.; Inorg. Chem. 1997, 36, 3096; Beneto, M.; Soto, L.; Garcia-Lozano, J.; Escriva, E.; Legros, J. -P.; Dahan, 
F.; J. Chem. Soc. Dalton Trans. 1991, 1057; Krupicka, E.; Lentz, A. Z.; Kristallogr. New Cryst. Struct. 2000, 215, 575; Habenschuss, M.; Gerstein, B. C.; J. Chem. Phys. 1974, 61, 852; Lin, K.-J.; Lii, K.-H.; Angew. Chem., Int. Ed. 1997, 36, 2076; Lai, S. F.; Cheng, C. Y.; Lin, K. J.; Chem. Commun. 2001, 1082; Spandl, J.; Brudgam, I.; Hartl, H.; Angew. Chem., Int. Ed. 2001, 40, 4018; Gutschke, S. O. H.; Molinier, M.; Powell, A. K.; Wood, P. T.; Angew. Chem., Int. Ed. 1997, 36, 991.

25. Yufit, D. S.; Price, D. J.; Howard, J. A. K.; Gutschke, S. O. H.; Powell, A. K.; Wood, P. T.; Chem. Commun. 1999, 1561; Braga, D.; Grepioni, F.; Chem. Commun. 1998, 911; Trombe, J.-C.; Sabadie, L.; Millet, P.; Solid State Sci. 2002, 4, 1199; Trombe, J.-C.; Petit, J.-F.; Gleizes, A.; Eur. J. Solid State Inorg. Chem. 1991, 28, 669; Robl, C.; Weiss, A.; Z. Anorg. Allg. Chem. 1987, 546, 161; Spandl, J.; Brudgam, I.; Hartl, H.; Z. Anorg. Allg. Chem. 2003, 629, 539; Hilbers, M.; Meiwald, M.; Mattes, R.; Z. Naturforsch., B: Chem. Sci. 1996, 51, 57; Maji, T. K.; Mostafa, G.; Sain, S.; Prasad, J. S.; Chaudhuri, N. R.; Cryst. Eng. Commun. 2001, 37, 1; Mukherjee, P. S.; Konar, S.; Zangrando, E.; Diaz, C.; Chaudhuri, N. R.; J. Chem. Soc., Dalton Trans. 2002, 3471; Manna, S. C.; Zangrando, E.; Ribas, J.; Chaudhuri, N. R.; Inorg. Chim. Acta 2005, 358, 4497; Ghosh, A. K.; Ghoshal, D.; Zangrando, E.; Ribas, J.; Chaudhuri, N. R.; Dalton Trans. 2006, 1554; Das, N.; Ghosh, A.; Arif, A. M.; Stang, P. J.; Inorg. Chem. 2005, 44, 7130; Crispini, A.; Pucci, D.; Aiello, I.; Ghedini, M.; Inorg. Chim. Acta 2000, 304, 219.

26. Deguenon, D.; Bernardelli, G.; Tuchagues, J. P.; Castan, P.; Inorg. Chem. 1990, 29, 3031; Castro, I.; Sletten, J.; Faus, J.; Julve, M.; Journaux, Y.; Lloret, F.; Alvarez, S.; Inorg. Chem. 1992, 31, 1889; Goncalves, N. S.; Santos, P. S.; Vencato, I.; Acta Crystallogr. 1996, C52, 622; Sletten, J.; Bjorsvik, O.; Acta. Chem. Scand. 1998, 52, 770; Dunitz, J. D.; Seiler, P.; Czechtizky, W.; Angew. Chem., Int. Ed. 2001, 40, 1779; Castro, I.; Calatayud, M. L.; Lloret, F.; Sletten J.; Julve, M.; J. Chem. Soc., Dalton Trans. 2002, 2397; Maji, T. K.; Konar, S.; Mostafa, G.; Zangrando, E.; Lu, T. H.; Chaudhuri, N. R.; J. Chem. Soc., Dalton Trans. 2003, 171; Maji, T. K.; Ghoshal, D.; Zangrando, E.; Ribas, J.; Chaudhuri, N. R.; CrystEngComm. 2004, 6, 623; Ghoshal, D.; Ghosh, A. K.; Ribas, J.; Mostafa, G.; Chaudhuri, N. R.; CrystEngComm. 2005, 7, 616; Wang, C. C.; Lin, H. W.; Yang, C. H.; Liao, C. H.; Lan, I. T.; Lee, G. H.; New J. Chem. 2004, 28, 180; Wang, C. C.; Yang, C. H.; Tseng, S. M.; Lee, G. H.; Chiang, Y. P.; Sheu, H. S.; Inorg. Chem. 2003, 42, 8294; Wang, C. C.; Yang, C. H.; Lee, G. H.; Inorg. Chem. 2002, 41, 1015; Wang, C. C.; Kuo, C. T.; Chou, P. T.; Lee, G. H.; Angew. Chem., Int. Ed. 2004, 43, 4507.

27. Castro, I.; Sletten, J.; Faus, J.; Julve, M.; J. Chem. Soc., Dalton Trans. 1992, 2271; Glick, M. D.; Downs, G. L.; Dahl, L. F.; Inorg. Chem. 1964, 3, 1712; Castan, P.; Deguenon, D.; Dahan, F.; Acta Crystallogr., Sect. C 1991, 47, 2656; Castro, I.; Sletten, J.; Glærum, L. K.; Lloret, F.; Faus, J.; Julve, M.; J. Chem. Soc., Dalton Trans. 1994, 2777; Sletten, J.; Bjørsvik, O.; Acta Chem. Scand. 1998, 32, 770; Deguenon, D.; Castan, P.; Dahan, F.; Acta Crystallogr., Sect. C 1991, 47, 433; Deguenon, D.; Bernardelli, G.; Tuchagues, J. P.; Castan, P.; Inorg. Chem. 1990, 29, 3031; Chen, Q.; Liu S.; Zubieta, J.; Inorg. Chim. Acta 1990, 175, 241; Wang, C. C.; Yang , C. H.;. Lee, G. H.; Inorg. Chem. 2002, 41, 1015.

28. Santos, P. S.; Sala, O.; Noda, L. K.; Gonçalves, N. S.; Spectrochim. Acta 2000, 56A, 1562.

29. de Oliveira, L. F. C.; Tese de Doutoramento, Universidade de São Paulo, Brasil, 1991.

30. Huheey, J.; Keiter, E. A.; Keiter, R. L.; Inorganic Chemistry: Principles of Structure and Reactivity, $4^{\text {th }}$ ed., Harper Collins: New York, 1993.

31. Fu, A.; Lu, J. Y.; Huang, X.; Li, J.; J. Alloys Compd. 2001, 89, 319.

32. Lam, C. K.; Mark, T. C. W.; Tetrahedron 2000, 56, 6657.

33. Mathew, S.; Paul, G.; Shivasankar, K.; Choudhury, A.; Rao, C. N. R.; J. Mol. Struct. 2002, 641, 263.

34. Dumestre, F.; Soula, B.; Galibert, A-M.; Fabré, P-L.; Bernardinelli, G.; Donnadieu, B.; Castan, P.; J. Chem. Soc., Dalton Trans. 1998, 4131.

35. Uçar, I.; Bulut, A.; Acta Cryst. E 2005, 61, m2730.
36. Köroglu, A.; Bulut, A.; Uçar, I.; Nichol, G. S.; Harrington, R. W.; Clegg, W.; Acta Cryst. C 2005, 61, o678.

37. Das, N.; Ghosh, A.; Arif-Stang, A. M.; Inorg. Chem. 2005, 44, 7130; Mathew, S.; Paul, G.; Shivasankar, K.; Choudhury, A.; Rao, C. N. R., J. Mol. Struct. 2002, 641, 263.

38. Bouayad, A.; Trombe, J-C.; Gleizes, A.; Inorg. Chim. Acta 1995, 230, 1.

39. Chen, Q.; Liu, S.; Zubieta, J.; Inorg. Chim. Acta 1990, 175, 269.

40. Fatiadi, A. J.; J. Am. Chem. Soc. 1978, 100, 2586.

41. Gonçalves, N. S.; Noda, L. K.; Neto, A. M. P.; Santos, P. S.; Mutarelli, S. R.; Sala, O.; J. Mol. Struc. 2002, 645, 185.

42. de Oliveira, L. F.; Mutarelli, S. R.; Gonçalves, N. S.; Santos, P. S.; Quim. Nova 1992, 15, 55.

43. Silva, M. R.; Dermat. Atual 1998, 4, 10.

44. Bigelow, R. W.; Freund, H. J.; J. Chem. Phys. 1986, 107, 159.

45. Gerecht, B.; Kämpchen, T.; Köhler, K.; Massa, W.; Offermann, G.; Schmidt, R. E.; Seitz, G.; Sutrisno, R.; Chem. Ber. 1984, 117, 2714.

46. Fabre, P. L.; Galibert, A. M.; Soula, B.; Dahan, F.; Castan, P.; J. Chem. Soc., Dalton Trans. 2001, 1529.

47. Fabre, P. L.; Pena, C.; Galibert, A. M.; Soula, B.; Bernadinelli, G.; Donnadieu, B.; Castan, P.; Can. J. Chem. 2000, 78, 280.

48. Pena, C.; Galibert, A. M.; Soula, B.; Fabre, P-L.; Bernadinelli, G.; Castan, P.; J. Am. Chem. Soc., Dalton Trans. 1998, 239.

49. Dumestre, F.; Soula, B.; Galibert, A.-M.; Fabré, P.-L.; Bernardinelli, G.; Donnadieu, B.; Castan, P.; J. Chem. Soc., Dalton Trans. 1998, 4131; Soula, B.; Galibert, A.-M.; Donnadieu, B.; Fabré, P.-L.; Inorg. Chim. Acta 2001, 324, 90; Teles, W. M.; Farani, R. A.; Maia, D. S.; Speziali, N. L.; Yoshida, M. I.; de Oliveira; L. F. C.; Machado, F. C.; J. Mol. Struct. 2006, 783, 52; Teles, W. M.; Farani, R. A.; Speziali, N. L.; Yoshida, M. I.; de Oliveira, L. F. C.; Machado, F. C.; Inorg. Chim. Acta 2006, 359, 3384.

50. Soula, B.; Galibert, A.-M.; Donnadieu, B.; Fabré, P.-L.; J. Chem. Soc., Dalton Trans. 2003, 2449.

51. Dumestre, F.; Soula, B.; Galibert, A. M.; Fabre, P. L.; Bernardinelli, G.; Donnadieu, B.; Castan, P.; J. Chem. Soc. Dalton Trans. 1998, 4131.

52. Soula, B.; Galibert, A.-M.; Donnadieu, B.; Fabre, P.-L.; J. Chem. Soc., Dalton Trans. 2003, 2449.

53. Kamat, P. V.; Fox, M. A.; Fatiadi, A. J.; J. Am. Chem. Soc. 1984, 106, 1191.

54. Kamat, P. V.; Fox, M. A.; J. Electroanal. Chem. 1983, 159, 49.

55. Tong, L.; Bi-Xian, P.; Dyes Pig. 1999, 43, 73.

56. Tran, K.; Scott, G. W.; Funk, D. J.; Moore, D. S.; J. Phys. Chem. US 1996, 100, 11863.

57. Lopes, J. G. S.; Farani, F. A.; de Oliveira, L. F. C.; Santos, P. S.,J. Raman Spec. 2006, 37, 142.

58. Sreejith, S.; Carol, P.; Chithraa, P.; Ajayaghosh, A.; J. Mater. Chem. 2008, 18, 264.

59. Oswald, B.; Patsenker, L.; Duschl, J.; Szmacinski, H.; Wolfbeis, O. S.; Terpetschnig, E.; Bioconjugate Chem. 1999, 10, 925.

60. Tatarets, A. L.; Fedyunyaeva, I. A.; Terpetschnig, E.; Patsenker, L. D.; Dyes Pigm. 2005, 64, 125.

61. Park, S.-Y.; Jun, K.; Oh, S. W.; Bull. Korean Chem. Soc. 2005, 26, 428.

62. Santos, P. F.; Reis, L. V.; Almeida, P.; Oliveira, A. S.; Vieira, L. F.; J. Photochem. Photobiol. A: Chem. 2003, 160, 159.

63. Neuse, E. W.; Green, B. R.; J. Org. Chem. 1974, 39, 3881.

64. Jiao, G. S.; Loudet, A.; Lee, H. B.; Kalinin, S.; Johansson, L. B-A.; Burgess, K.; Tedrahedron 2003, 59, 3109.

65. Block, M. A. B.; Khan, A.; Hecht; J. Org. Chem. 2004, 69, 184.

66. Tatarets, A. L.; Fedyunyayeva, I. A.; Dyubko, T. S.; Povrozin, Y. A.; Doroshenko, A. O.; Terpetschnig, E. A.; Patsenker, L. D.; Anal. Chim. Acta 2006, 570, 214.

67. Ajayaghosh, A.; Acc. Chem. Res. 2005, 38, 449.

68. Prodi, L.; Ballardini, R.; Gandolfi, M. T.; Roversi, R.; J. Photochem. Photobiol. A 2000, 136, 49; Momotake, A.; Arai, T.; Tetrahedron Lett. 
2003, 44, 7277; Cha, N. R.; Moon, S. Y.; Chang, S.-K., Tetrahedron Lett. 2003, 44, 8265; Nakahara, Y.; Kida, T.; Nakatsuji, Y.; Akashi, M.; J. Org. Chem. 2004, 69, 4403; Kawakami, J.; Kimura, H.; Nagaki, M.; Kitahara, H.; Ito, S.; J. Photochem. Photobiol. A 2004, 161, 141.

69. Huston, M. E.; Haider, K. W.; Czarnik, A. W.; J. Am. Chem. Soc 1988, 110, 4460; Fabbrizzi, L.; Lichelli, M.; Pallavicini, P.; Perotti, A.; Sacchi, D.; Angew. Chem., Int. Ed. 1994, 33, 1975; Kawakami, J.; Komai, Y.; Ito, S.; Chem. Lett. 1996, 617; Fabbrizzi, L.; Lichelli, M.; Pallavicini, P.; Perotti, A.; Taglietti, D.; Sacchi, D.; Chem.-Eur. J. 1996, 2, 167; Habata, Y.; Fukuda, Y.; Akabori, S.; Bradshaw, J. S.; J. Chem. Soc., Perkin Trans. 1, 2002, 865; Herranz, M. A.; Colonna, B.; Echegoyen, L.; Proc. Natl. Acad. Sci. U.S.A. 2002, 99, 5040; Hayashita, T.; Qing, D.; Minagawa, M.; Lee, J. C.; Ku, C. H.; Teramae, N.; Chem. Commun. 2003, 2160.

70. Law, K. Y.; J. Phys. Chem. 1989, 93, 5925; Das, S.; Kamat, P. V.; De la Barre, B.; Thomas, K. G.; Ajayaghosh, A.; George, M. V. J.; Phys. Chem. 1992, 96, 10327; Das, S.; Thomas, K. G.; George, M. V.; Kamat, P. V.; J. Chem. Soc. Faraday Trans. 1992, 88, 3419; Chen, H.; Herkstroeter, W. G.; Perlstein, J.; Law, K.-Y.; Whitten, D. G.; J. Phys. Chem. 1994, 98, 5138; Das, S.; Thomas, K. G.; Ramanathan, R.; Kamat, P. V.; George, M. V. J., Phys. Chem. 1993, 97, 13625.

71. Beer, P. D.; Danks, J. P.; Hesek, D.; McAleer, J. F.; J. Chem. Soc., Chem. Commun. 1993, 1735; Beer, P. D.; Endeavour, New Ser. 1992, 16, 182; Beer, P. D.; Kocian, O.; Mortimer, R. J.; Ridgway, C.; Analyst 1992, 117,1247
72. Kamat, P. V.; Das, S.; Thomas, K. G.; George, M. V.; J. Phys. Chem. 1992, 96, 195.

73. Silva, C. E.; Diniz, R.; Rodrigues, B. L.; de Oliveira, L. F. C., J. Mole. Struc., no prelo.

74. Law, K. Y.; J. Phys. Chem. 1995, 99, 9818.

75. Stokes, R. J.; Ingram, A.; Gallagher, J.; Armstrong, D. R.; Smith, W. E.; Graham, D.; Chem. Commun. 2008, 567.

76. Honeybourne, C. L.; J. Mater. Chem. 1999, 9, 2241; Bigelow, R. W.; Freund, H. J.; Chem. Phys. 1986, 107, 159.

77. Lunelli, B.; Monari, M.; Bottoni, A.; J. Phys. Chem. A 2001, 105, 2257.

78. Busetti, V.; Lunelli, B.; J. Phys. Chem. 1986, 90, 2052.

79. Pena, C.; Galibert, A. M.; Soula, B.; Fabre, P-L.; Bernadinelli, G.; Castan, P.; J. Am. Chem. Soc., Dalton Trans. 1998, 239.

80. Klewe, B.; Acta Chem. Scand. 1971, 25, 1975; de Oliveira, V. E.; Dissertação de Mestrado, Universidade Federal de Juiz de Fora, Brasil, 2007.

81. de Oliveira, V. E.; Freitas, M. C. R.; Diniz, R.; Yoshida, M. I.; Speziali, N. L.; Edwards, H. G. M.; de Oliveira, L. F. C.; J. Mol. Struc. 2008, $881,57$.

82. Galibert, A. M.; Soula, B.; Donnadieu, B.; Fabre, P. L.; Inorg. Chim. Acta 2001, 313, 160; Farnia, G.; Lunelli, B.; Marcuzzi, F.; Sandonà, G.; J. Electr. Chem. 1996, 404, 261. 